\title{
Artesunate suppresses oxidative and inflammatory processes by activating Nrf2 and ROS-dependent p38 MAPK and protects against cerebral ischemia-reperfusion injury
}

\author{
HUI LU ${ }^{1}$, BINCHENG WANG $^{2}$, NINGNING CUI ${ }^{1}$ and YANCHUN ZHANG ${ }^{1}$ \\ ${ }^{1}$ Department of Neurology, Cangzhou Central Hospital, Cangzhou, Hebei 060000; \\ ${ }^{2}$ Department of Neurology, Xuan Wu Hospital, Beijing 100053, P.R. China
}

Received June 14, 2016; Accepted April 25, 2017

DOI: $10.3892 / \mathrm{mmr} .2018 .8666$

\begin{abstract}
Artesunate is a semi-synthetic derivative of artemisinin that is used in the treatment of patients with malaria. Artesunate has also been reported to exert immune-regulatory, antitumor, hepatoprotective, anti-inflammatory and smooth muscle relaxing functions. The present study aimed to investigate the putative protective effects of artesunate against cerebral ischemia/reperfusion injury (CIRI), and to elucidate the molecular mechanisms underlying its effects. A CIRI mouse model was created via middle cerebral artery occlusion for $2 \mathrm{~h}$, followed by $22 \mathrm{~h}$ of reperfusion. Mice were treated with $10-40 \mathrm{mg} / \mathrm{kg}$ artesunate. The present results demonstrated that treatment with artesunate significantly reduced the cerebral infarct volume and potentiated the recovery of neurological function in CIRI mice. Oxidative stress and inflammation markers were revealed to be significantly downregulated following treatment with artesunate in CIRI mice. Furthermore, artesunate was demonstrated to activate nuclear factor erythroid 2-related factor 2 (Nrf2), inhibit caspase-3 activity, reduce the apoptosis regulator BAX/apoptosis regulator Bcl-2 expression ratio and suppress the phosphorylation of the mitogen-activated protein kinase (MAPK) p38 in CIRI mice. In conclusion, the present findings suggested that artesunate may exert protective effects against CIRI through the suppression of oxidative and inflammatory processes, via activating Nrf2 and downregulating ROS-dependent p38 MAPK in mice.
\end{abstract}

Correspondence to: Dr Hui Lu, Department of Neurology, Cangzhou Central Hospital, Thomas Regency East 3-1-2403, Cangzhou, Hebei 060000, P.R. China

E-mail: luhuihb@126.com

Key words: artesunate, cerebral ischemia-reperfusion injury, oxidation, inflammation, nuclear factor erythroid 2-related factor 2, mitogen-activated protein kinase p38

\section{Introduction}

Cerebrovascular diseases are common conditions caused by impairments in the oxygen supply to the brain, among which stroke is characterized by a high incidence rate, and is one of the most common causes of morbidity and mortality worldwide $(1,2)$. Comorbidities associated with stroke include visual impairments, loss of speech, paralysis and mental disorders (3). Stroke severely degrades the health and quality of life of surviving patients, and it poses a heavy economic and psychological burden for the patients and their families, and a major health concern for society (3).

Stroke is an acute disorder caused by brain hypoxia or ischemia, as a result of blood vessel blockage or cerebral blood vessel rupture and subsequent hemorrhage (4). Strokes may thus be divided into ischemic strokes and hemorrhagic strokes, with ischemic strokes accounting for $\sim 87 \%$ of all stroke cases. Cerebral ischemia can rapidly cause the necrosis of cerebral tissue in the ischemic nucleus, followed by tardive neuronal death in ischemic and surrounding tissues, which ultimately results in brain damage (5). The primary challenge to the treatment of ischemic stroke is the short time window for successful intervention; as a result, a large number of stroke victims fail to be treated in a timely and effective manner, with severe consequences to the restoration of brain function (6).

Disturbances in energy homeostasis are among the main causes of cerebral ischemia/reperfusion injury (CIRI) (7). Oxidative stress is involved in CIRI, as the dysregulation of mitochondrial oxidative phosphorylation leads to a decrease in ATP production and an increase in reactive oxygen species (ROS) generation (7). In addition to the decrease in ATP levels and the increased production of free radicals, which can directly damage cellular components, adaptive regulation mechanisms of CIRI also participate in the molecular mechanisms underlying the pathology of the disorder (8).

The area of the ischemic event is composed of a necrotic area in the infarct focus, and a surrounding ischemic penumbra (9). Cells in the center of the infarction are largely necrotic, whereas the ischemic penumbra is mainly characterized by cellular apoptosis; however, penumbral cells may be salvaged following reperfusion (9). Cellular apoptosis is 
a physiological process that can be activated by internal and external factors, and is regulated by several genes, enzymes and signal transduction pathways (9). The mitogen-activated protein kinase (MAPK) cascade communicates signals from the cell surface to the nucleus and is involved in numerous physiological cellular processes, including adaptation, proliferation, differentiation, survival and apoptosis (10). Therefore, the implication of the MAPK pathway in the molecular mechanisms of CIRI has garnered considerable attention.

p38 MAPK is a critical member of the MAPK family, which can be phosphorylated in response to various extracellular stimuli, thus activating the MAPK signaling pathway (11). P38 MAPK has been implicated in the regulation of inflammatory responses, and has been associated with cellular differentiation, cell cycle progression and apoptosis (12). During CIRI, p38/MAPK signaling has been reported to be activated, as p38 MAPK has been demonstrated to translocate from the cytoplasm into the nucleus, activate downstream kinases and transcription factors, and modulate the expression of apoptosis-associated genes, thus promoting cellular apoptosis (13).

The extracts of the Chinese medicinal herb Artemisia carvifolia have demonstrated diverse pharmacological properties, including immune-regulatory, anti-inflammatory, antitumor and anti-angiogenetic effects (14). Artesunate (dihydroartemisinin 1,2 $\alpha$ succinic acid monoester) is a semi-synthetic sesquiterpene lactonic derivative of the antimalarial drug artemisinin, which is one of the main active ingredients of Artemisia carvifolia (15). Artesunate is a novel antimalarial drug, characterized by high efficacy, fast action, low toxicity and low tolerance (15). In addition, artesunate has exhibited anti-tumor activity accompanied by low toxicity and has been used successfully for the treatment of patients with metastatic melanoma in clinical practice (15). Previous studies have reported that artesunate increased the intracellular production of oxygen free radicals, and intervened in nuclear factor (NF)-kB- and phosphatidylinositol-4,5-bisphosphate 3-kinase/Akt-mediated signaling pathways, thus causing DNA damage, interfering with cell cycle regulation, and preventing metastasis and multidrug resistance (15-17). Therefore, the present study investigated the effects of artesunate during CIRI and the possible molecular mechanisms underlying its actions.

\section{Materials and methods}

Animals and experimental protocol. The experimental procedures used in the present study were reviewed and approved by the Animal Experiment Ethics Committee of Cangzhou Central Hospital (Cangzhou, China). Male adult Kunming mice (weight, 21-23 g; age, 6-7 weeks; $n=30$ ) were purchased from the Experimental Animal Center of Cangzhou Central Hospital and were allowed to acclimate to lab conditions for 1 week prior to the commencement of experiments. Mice were housed at a temperature of $21-23^{\circ} \mathrm{C}$ and $55-60 \%$ relative humidity, under a 12-h light/dark cycle, with free access to food and water.

Mice were randomly assigned to the following 5 groups: Sham-operated group $(n=6)$; CIRI model group $(n=6)$, mice

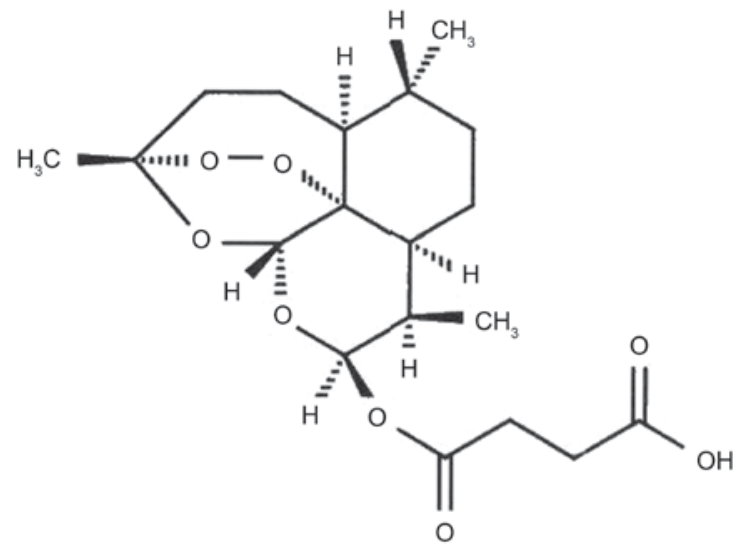

Figure 1. Chemical structure of artesunate.

were subjected to middle cerebral artery occlusion for $2 \mathrm{~h}$, followed by $22 \mathrm{~h}$ reperfusion, and treated with normal saline for 7 days; CIRI + low artesunate group $(n=6)$, CIRI mice were treated with $10 \mathrm{mg} / \mathrm{kg} /$ day artesunate (intraperitoneal administration, Sigma-Aldrich; Merck KGaA, Darmstadt, Germany) for 7 days; CIRI + medium artesunate group $(n=6)$, CIRI mice were treated with $20 \mathrm{mg} / \mathrm{kg} / \mathrm{day}$ artesunate (intraperitoneal administration) for 7 days; and CIRI + high artesunate group $(\mathrm{n}=6)$, CIRI mice were treated with $40 \mathrm{mg} / \mathrm{kg} / \mathrm{day}$ artesunate (intraperitoneal administration) for 7 days.

Establishment of CIRI mouse model. Mice were anesthetized with $35 \mathrm{mg} / \mathrm{kg}$ sodium pentobarbital. A midline incision was conducted, and the right common, external and internal carotid arteries were exposed. The right common and external carotid arteries were ligated using 6-0 sutures, and the internal carotid artery was subsequently occluded using a clamp. A silicone-coated 6-0 nylon monofilament was inserted into the internal carotid artery from a small incision on the right common carotid artery, until the tip blocked the origin of the middle cerebral artery. The surgical incisions were closed with $6-0$ sutures, mice were maintained at $37^{\circ} \mathrm{C}$ for $2 \mathrm{~h}$, and the clamp was subsequently removed for reperfusion. Subsequently, mice were treated with artesunate.

Neurological evaluation. Following treatment with artesunate, the neurological function of mice was examined as previously described (18). The following grading system was used for evaluation: 0 , No neural function deficit; 1 , left forepaw weakness; 2 , constantly turning left; 3 , mouse falling to the contralateral side; 4 , mouse unable to walk spontaneously or comatose.

Histological examination. Following treatment with artesunate, the brains were isolated and sectioned into $4 \mathrm{~mm}$ coronal slices. To evaluate cerebral infarct volume, the brain tissue sections were incubated with $1 \%$ 2,3,5-triphenyl-tetrazolium chloride for $30 \mathrm{~min}$ at $37^{\circ} \mathrm{C}$ in the dark and then fixed with $10 \%$ formalin at room temperature for $24 \mathrm{~h}$. Photographs of stained brain tissue samples were captured using a digital camera (Nikon Corporation, Tokyo, Japan) and analyzed using ImageJ software (version 3.1; National Institutes of Health, Bethesda, MD, USA). For histopathological examination, 
A

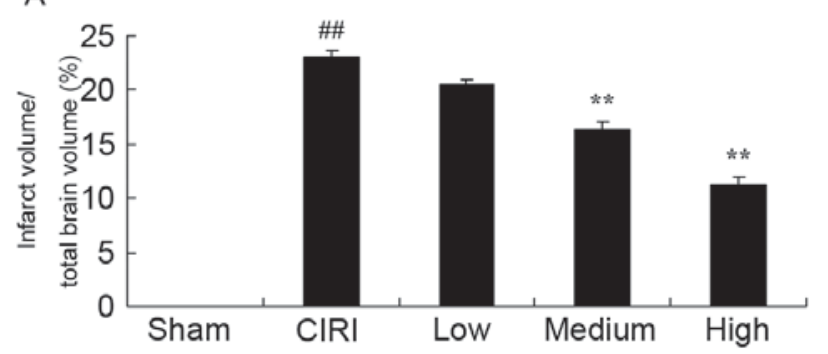

B

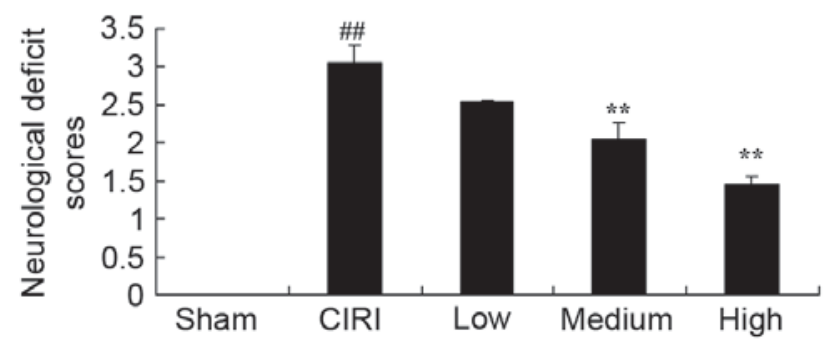

Figure 2. Treatment with artesunate reduces cerebral infarct volume and protects neurological function in CIRI mice. (A) Cerebral infarct volumes were significantly reduced in CIRI mice following treatment with artesunate. (B) Treatment with artesunate prevented CIRI-associated deficits in neurological function. Sham, sham-operated group; CIRI, CIRI model group; Low, CIRI + $10 \mathrm{mg} / \mathrm{kg}$ artesunate for 7 days group; Medium, CIRI + $20 \mathrm{mg} / \mathrm{kg}$ artesunate for 7 days group; High, CIRI $+40 \mathrm{mg} / \mathrm{kg}$ artesunate for 7 days group. ${ }^{\# \#} \mathrm{P}<0.01 \mathrm{vs}$. Sham; ${ }^{* *} \mathrm{P}<0.01$ vs. CIRI. CIRI, cerebral ischemia/reperfusion injury.

hippocampi were isolated, fixed in $10 \%$ formalin at room temperature for $24 \mathrm{~h}$, embedded in paraffin and sliced into 4- $\mu \mathrm{m}$ sections. Subsequently, tissue sections were deparaffinized and stained with hematoxylin and eosin (H\&E) at room temperature for 10-15 min and observed with a fluorescence microscope (magnification, x10; Zeiss GmbH, Jena, Germany).

Evaluation of proinflammatory factors, oxidative stress and caspase-3 activity. Blood samples were collected and the supernatants were isolated after $2,000 \mathrm{x} \mathrm{g}$ for $10 \mathrm{~min}$ at $4^{\circ} \mathrm{C}$ to measure the activity of interleukin (IL)-1 $\beta$ (cat. no. H002), IL-6 (cat. no. H007), IL-10 (cat. no. H009), tumor necrosis factor (TNF)- $\alpha$ (cat. no. H052), glutathione (GSH; cat. no. A006-2) and superoxide dismutase (SOD; cat. no. A001-2) using ELISA kits (Nanjing Jiancheng Bioengineering Institute, Nanjing, China). The absorbance of the samples was measured at $450 \mathrm{~nm}$ using a Model 680 microplate reader (Bio-Rad Laboratories, Inc., Hercules, CA, USA). ROS production was evaluated using a Reactive Oxygen Species Assay kit (Beyotime Institute of Biotechnology, Haimen, China) with a Model 680 microplate reader (Bio-Rad Laboratories, Inc.), using an excitation wavelength of $488 \mathrm{~nm}$ and an emission wavelength of $525 \mathrm{~nm}$. Caspase-3 activity was measured using a Caspase-3 Activity Assay kit (Beyotime Institute of Biotechnology). The absorbance of the samples was measured at $405 \mathrm{~nm}$ using a Model 680 microplate reader (Bio-Rad Laboratories, Inc.)

Western blot analysis. The hippocampus and cortex of the injured hemisphere were isolated and homogenized in cold lysis buffer containing phenylmethylsulfonyl fluoride (Beyotime Institute of Biotechnology) at $4^{\circ} \mathrm{C}$ for $30 \mathrm{~min}$. The supernatants were collected following centrifugation $12,000 \mathrm{x} \mathrm{g}$ for $10 \mathrm{~min}$ at $4^{\circ} \mathrm{C}$ and protein concentration was measured using a bicinchoninic acid protein assay kit (Beyotime Institute of Biotechnology). Equal amounts of extracted protein samples $(50 \mu \mathrm{g})$ were separated by $10 \%$ SDS-PAGE and transferred onto polyvinylidene difluoride membranes. Membranes were blocked with $5 \%$ milk in TBST at room temperature for $1 \mathrm{~h}$ and incubated overnight at $4^{\circ} \mathrm{C}$ with the following primary antibodies: Anti- nuclear factor erythroid 2-related factor 2(Nrf2; 1:500; cat.no. sc-722; Santa Cruz Biotechnology, Inc.), anti-apoptosis regulator Bcl-2(Bcl-2; 1:500; cat.no. sc-783; Santa Cruz Biotechnology, Inc.), anti-apoptosis regulator BAX
(Bax; 1:500; cat. no. sc-6236; Santa Cruz Biotechnology, Inc.), anti-phosphorylated (p)-p38 MAPK (1:500; cat. no. sc-7975-R; Santa Cruz Biotechnology, Inc.) and anti-GAPDH (1:1,000; cat.no. sc-367714; Santa Cruz Biotechnology, Inc.). Membranes were washed with TBS containing Tween-20 (0.1\%) and then incubated with horseradish peroxidase-conjugated anti-rabbit $\operatorname{IgG}(\mathrm{H}+\mathrm{L})$, Biotinylated Antibody (1:5,000; cat. no. 14708; Cell Signaling Technology, Inc.) at $37^{\circ} \mathrm{C}$ for $2 \mathrm{~h}$. Protein bands were visualized using an enhanced chemiluminescence system (Beyotime Institute of Biotechnology) and blots were semi-quantified using ImageJ software version 3.1 (National Institutes of Health).

Statistical analysis. All data are expressed as the mean \pm standard deviation $(n=3)$. Statistical analysis was performed with SPSS 17.0 software (SPSS, Inc., Chicago, IL, USA). The statistical significance of the differences between groups was assessed using one-way analysis of variance with Tukey post hoc test. $\mathrm{P}<0.05$ was considered to indicate a statistically significant difference.

\section{Results}

Treatment with artesunate reduces cerebral infarct volume and protects neurological function in CIRI mice. The chemical structure of artesunate is presented in Fig. 1. The effects of artesunate on cerebral infarct volume and its putative protective effects against neurological function impairments were investigated in mice following CIRI. As demonstrated in Fig. 2A, cerebral infarct volumes in mice from the CIRI model group were significantly larger compared with mice from the sham group, and their neurological function was significantly impaired (Fig. 2B). However, following treatment with 20 or $40 \mathrm{mg} / \mathrm{kg}$ artesunate, the cerebral infarct volume in CIRI mice was significantly reduced compared with untreated mice from the CIRI model group (Fig. 2A). In addition, treatment with medium and high doses of artesunate was revealed to significantly protect mice from CIRI-associated neurological deficits (Fig. 2B).

Treatment with artesunate alleviates cerebral histopathological alterations in CIRI mice. In order to investigate the putative protective effects of artesunate against CIRI-induced histopathological alterations in cerebral tissue, hippocampi 


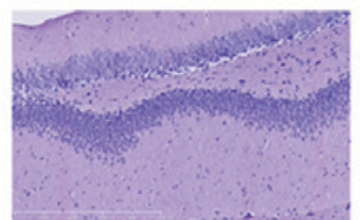

Sham

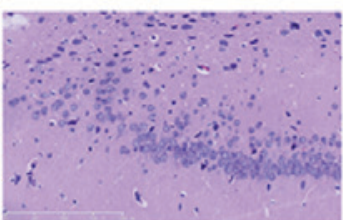

CIRI

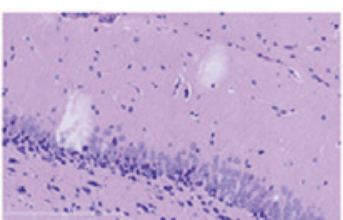

Low

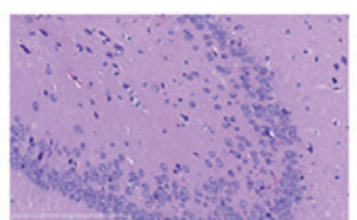

Medium

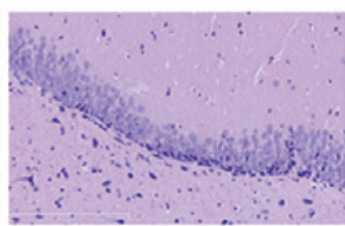

High

Figure 3. Treatment with artesunate alleviates cerebral histopathological alterations in CIRI mice. Hippocampal tissue samples were isolated and histological examination was performed following staining with hematoxylin and eosin (magnification, x10). Sham, sham-operated group; CIRI, CIRI model group; Low, CIRI $+10 \mathrm{mg} / \mathrm{kg}$ artesunate for 7 days group; Medium, CIRI + $20 \mathrm{mg} / \mathrm{kg}$ artesunate for 7 days group; High, CIRI + $40 \mathrm{mg} / \mathrm{kg}$ artesunate for $7 \mathrm{days}$ group. CIRI, cerebral ischemia/reperfusion injury.

A

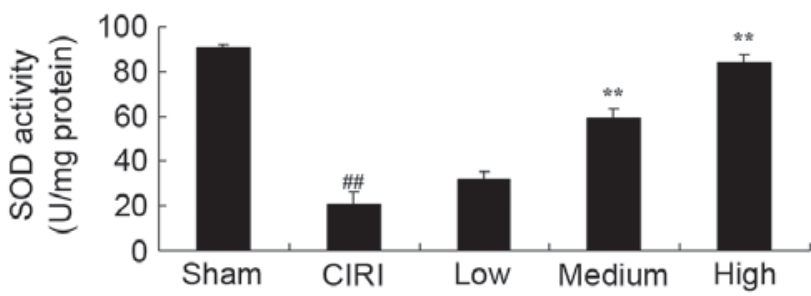

B

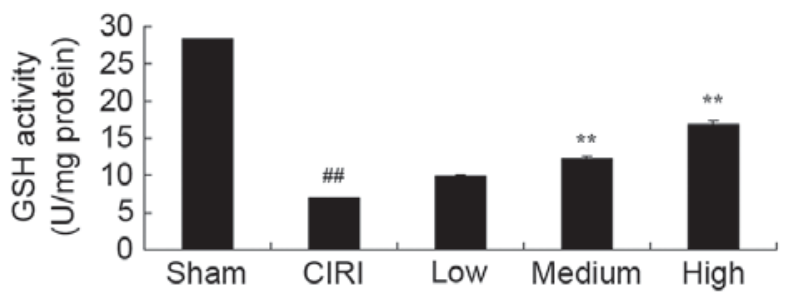

Figure 4. Treatment with artesunate protects endogenous antioxidant activity in CIRI mice. Treatment with artesunate counteracted the CIRI-associated suppression of (A) SOD and (B) GSH activity. Sham, sham-operated group; CIRI, CIRI model group; Low, CIRI + 10 mg/kg artesunate for 7 days group; Medium, CIRI $+20 \mathrm{mg} / \mathrm{kg}$ artesunate for 7 days group; High, CIRI $+40 \mathrm{mg} / \mathrm{kg}$ artesunate for 7 days group. ${ }^{\# \#} \mathrm{P}<0.01$ vs. Sham; ${ }^{* *} \mathrm{P}<0.01 \mathrm{vs}$. CIRI. CIRI, cerebral ischemia/reperfusion injury; SOD, superoxide dismutase; GSH, glutathione.

were isolated and stained with H\&E. As presented in Fig. 3, mice from the CIRI model group exhibited severe histological damage compared with mice from the sham group. Treatment with 20 or $40 \mathrm{mg} / \mathrm{kg}$ artesunate was demonstrated to markedly attenuate the CIRI-associated histopathological alterations in hippocampal tissue compared with tissue samples from untreated CIRI mice (Fig. 3).

Treatment with artesunate protects endogenous antioxidant activity in CIRI mice. In order to investigate the antioxidative effects of artesunate during CIRI, the activity of the endogenous antioxidants SOD and GSH were assessed using ELISA kits. Mice from the CIRI model group exhibited significantly reduced SOD and GSH activity compared with mice from the sham group (Fig. 4A and B). Notably, treatment with 20 or $40 \mathrm{mg} / \mathrm{kg}$ artesunate was revealed to restore the activity of SOD and GSH compared with untreated CIRI mice (Fig. 4A and B).

Treatment with artesunate suppresses ROS production in CIRI mice. In order to further investigate the effects of artesunate on oxidative stress during CIRI, ROS production was assessed. The present results demonstrated that ROS production was significantly potentiated in CIRI mice (Fig. 5). However, following treatment with 20 or $40 \mathrm{mg} / \mathrm{kg}$ artesunate, ROS production was significantly suppressed compared with untreated mice from the CIRI model group (Fig. 5).

Treatment with artesunate suppresses inflammatory responses in CIRImice. In order to evaluate the putative anti-inflammatory properties of artesunate during CIRI, ELISA kits were used to assess the activity of the proinflammatory cytokines IL-1 $\beta$, IL- 6 and TNF- $\alpha$, and of the anti-inflammatory cytokine

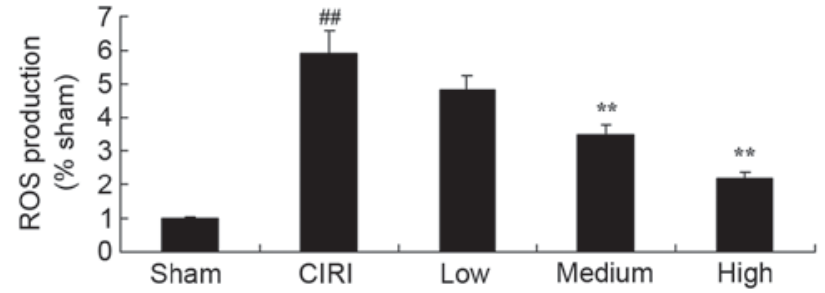

Figure 5. Treatment with artesunate suppresses ROS production in CIRI mice. ROS production was significantly enhanced during CIRI, whereas it was significantly downregulated following treatment with artesunate. Sham, sham-operated group; CIRI, CIRI model group; Low, CIRI $+10 \mathrm{mg} / \mathrm{kg}$ artesunate for 7 days group; Medium, CIRI $+20 \mathrm{mg} / \mathrm{kg}$ artesunate for 7 days group; High, CIRI + $40 \mathrm{mg} / \mathrm{kg}$ artesunate for 7 days group. ${ }^{\#} \mathrm{P}<0.01 \mathrm{vs}$. Sham; ${ }^{* *} \mathrm{P}<0.01$ vs. CIRI. CIRI, cerebral ischemia/reperfusion injury; ROS, reactive oxygen species.

IL-10. In CIRI mice, the activity of TNF- $\alpha$, IL-1 $\beta$ and IL-6 was significantly enhanced, whereas the activity of IL-10 was significantly suppressed compared with in sham-operated mice (Fig. 6A-D). Following treatment with 20 or $40 \mathrm{mg} / \mathrm{kg}$ artesunate, TNF- $\alpha$, IL-1 $\beta$ and IL-6 activity was significantly reduced, whereas the activity of IL-10 was significantly enhanced compared with untreated mice from the CIRI model group (Fig. 6A-D).

Treatment with artesunate prevents caspase-3 activation in CIRI mice. In order to investigate the molecular mechanisms underlying the protective effects of artesunate during CIRI, the activity of the proapoptotic caspase-3 was assessed. As revealed in Fig. 7, caspase-3 activity was significantly increased in mice from the CIRI model group compared with the sham-operated mice. Notably, following treatment 
A

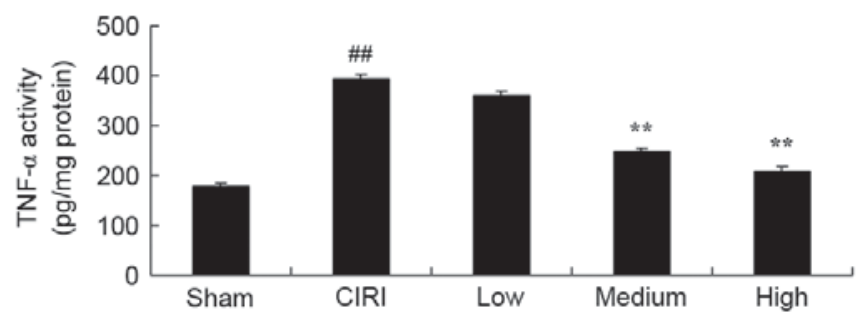

C

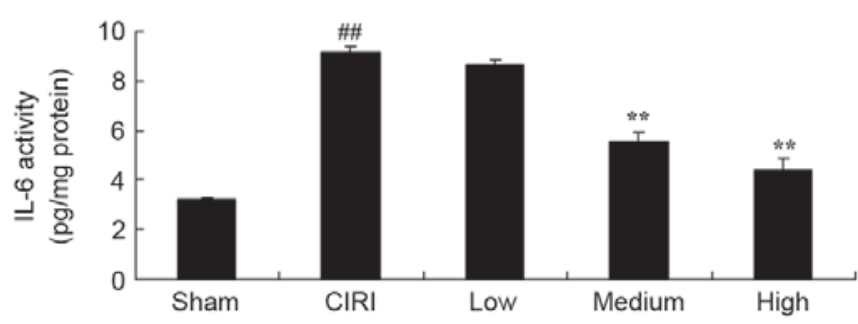

B

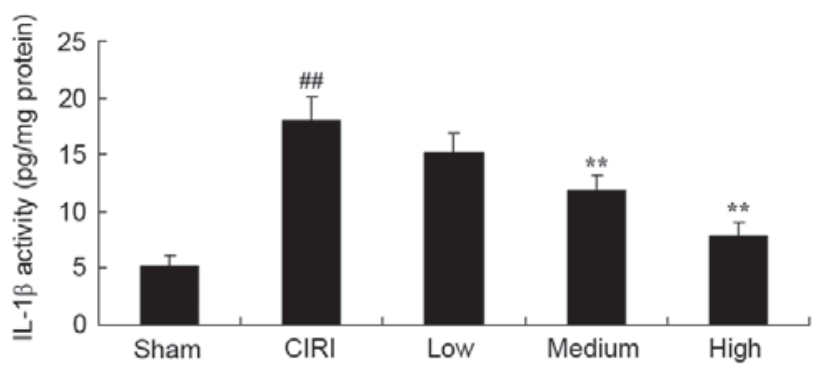

D

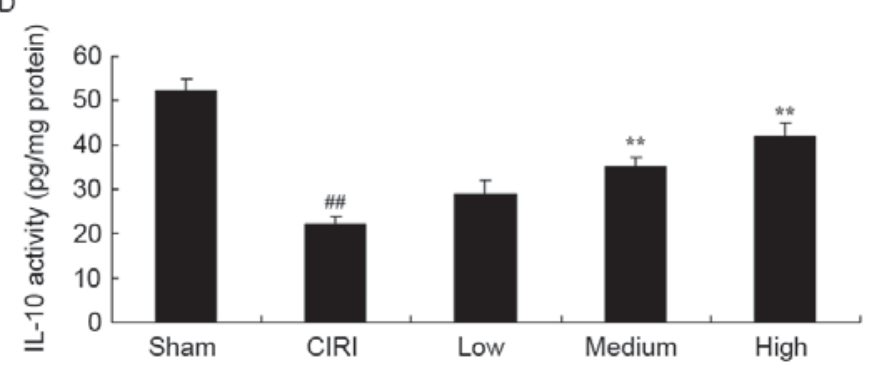

Figure 6. Treatment with artesunate suppresses inflammatory responses in CIRI mice. Following treatment with artesunate the activity of (A) TNF- $\alpha$, (B) IL-1 $\beta$ and (C) IL-6 was inhibited, whereas the activity of (D) IL-10 was enhanced. Sham, sham-operated group; CIRI, CIRI model group; Low, CIRI + 10 mg/kg artesunate for 7 days group; Medium, CIRI $+20 \mathrm{mg} / \mathrm{kg}$ artesunate for 7 days group; High, CIRI $+40 \mathrm{mg} / \mathrm{kg}$ artesunate for 7 days group. ${ }^{\# \prime} \mathrm{P}<0.01 \mathrm{vs}$. Sham; ${ }^{* *} \mathrm{P}<0.01$ vs. CIRI. CIRI, cerebral ischemia/reperfusion injury; TNF, tumor necrosis factor; IL, interleukin.

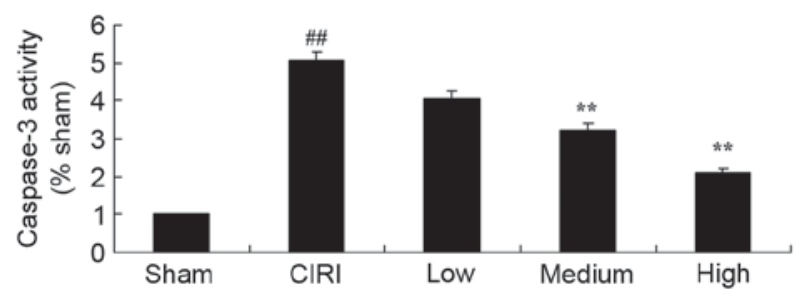

Figure 7. Treatment with artesunate prevents caspase-3 activation in CIRI mice. Caspase-3 activity was significantly upregulated in CIRI mice, whereas it was significantly suppressed following treatment with artesunate. Sham, sham-operated group; CIRI, CIRI model group; Low, CIRI + $10 \mathrm{mg} / \mathrm{kg}$ artesunate for 7 days group; Medium, CIRI $+20 \mathrm{mg} / \mathrm{kg}$ artesunate for 7 days group; High, CIRI + $40 \mathrm{mg} / \mathrm{kg}$ artesunate for 7 days group. ${ }^{\#} \mathrm{P}<0.01 \mathrm{vs}$. Sham; ${ }^{* *} \mathrm{P}<0.01$ vs. CIRI. CIRI, cerebral ischemia/reperfusion injury.

with artesunate, the activation of caspase- 3 was significantly suppressed compared with untreated CIRI mice (Fig. 7).

Treatment with artesunate restores Nrf2 protein expression in CIRI mice. To further investigate the protective mechanisms implicated in the effects of artesunate during CIRI, western blot analysis was used to assess the protein expression levels of the transcription factor Nrf2 (Fig. 8A). As demonstrated in Fig. 8B, Nrf2 protein expression was significantly downregulated in CIRI mice compared with mice from the sham group. Conversely, treatment with 20 or $40 \mathrm{mg} / \mathrm{kg}$ artesunate was revealed to significantly upregulate Nrf2 protein expression in CIRI mice compared with untreated mice from the CIRI model group (Fig. 8B).

Treatment with artesunate restores the Bax/Bcl-2 expression ratio in CIRI mice. To further explore the anti-apoptotic effects of artesunate during CIRI, the protein expression levels of the proapoptotic factor Bax and the antiapoptotic factor Bcl-2 were assessed using western blot analysis (Fig. 8A). The present results demonstrated that the $\mathrm{Bax} / \mathrm{Bcl}-2$ protein expression ratio was significantly increased in CIRI mice compared with in sham-operated mice (Fig. 8C). Following treatment with artesunate $(20$ or $40 \mathrm{mg} / \mathrm{kg}$ ), the $\mathrm{Bax} / \mathrm{Bcl}-2$ protein expression ratio was significantly reduced compared with untreated mice from the CIRI model group (Fig. 8C).

Treatment with artesunate suppresses p38 MAPK phosphorylation in CIRI mice. In order to investigate the involvement of the MAPK signaling pathway in the effects of artesunate during CIRI, the protein expression levels of p-p38 MAPK were assessed using western blot analysis (Fig. 8A). The present results revealed that the protein expression levels of $\mathrm{p}-\mathrm{p} 38$ MAPK were significantly upregulated in CIRI mice compared with in mice from the sham group (Fig. 8D). Following treatment with 20 or $40 \mathrm{mg} / \mathrm{kg}$ artesunate, $\mathrm{p}$-p38 protein expression levels in CIRI mice were significantly reduced compared with untreated mice from the CIRI model group (Fig. 8D).

\section{Discussion}

Cerebrovascular disease is also known as cerebrovascular accident or stroke, and is a common disease of the cerebral circulation with a high incidence rate (19). According to the third national survey of mortality causes released by the Ministry of Health in 2012, 136.64 out of 100,000 patients with stroke succumbed in China, raising the mortality of strokes to more than that of malignant tumors (20). In China, stroke has become the disease with the highest mortality rate (21). Thrombolysis, angioplasty, rehabilitation and other therapies, as well as early intervention, can restore cerebral blood supply 
A

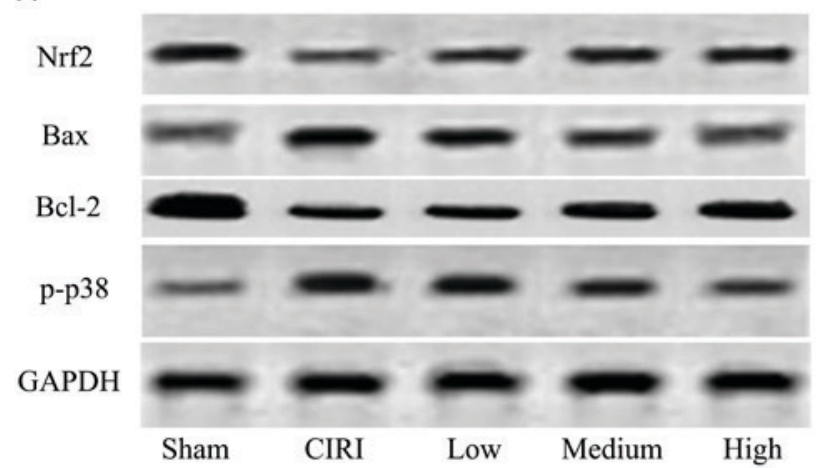

B

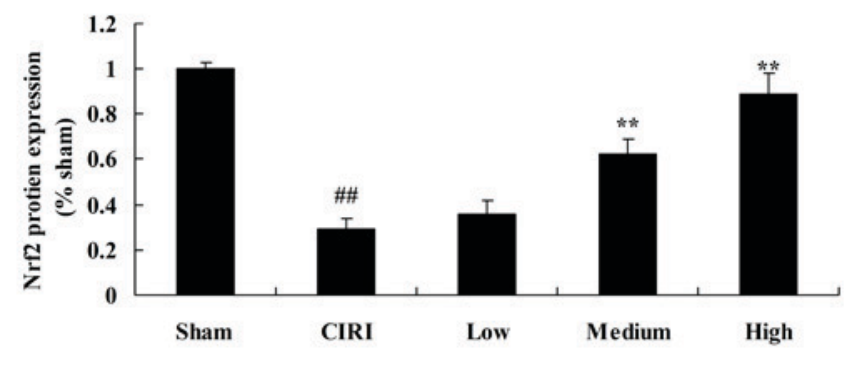

D

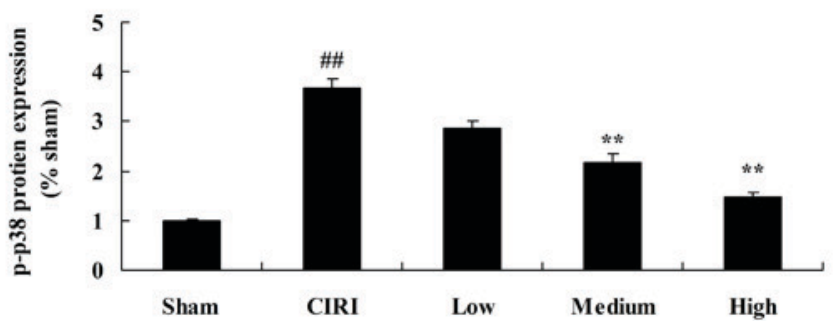

Figure 8. Treatment with artesunate restores Nrf2 protein expression and the Bax/Bcl-2 ratio, and suppresses p38 MAPK phosphorylation in CIRI mice. (A) Protein expression was detected using western blot analysis. (B) Blots were semi-quantified and statistical analysis demonstrated that treatment of CIRI mice with artesunate prevented the CIRI-induced downregulation of Nrf2 protein expression. (C) Statistical analysis demonstrated that treatment of CIRI mice with artesunate prevented the CIRI-induced increase in the Bax/Bcl-2 protein expression ratio. (D) Statistical analysis demonstrated that treatment of CIRI mice with artesunate prevented the CIRI-induced upregulation of p-p38 MAPK protein expression. Sham, sham-operated group; CIRI, CIRI model group; Low, CIRI $+10 \mathrm{mg} / \mathrm{kg}$ artesunate for 7 days group; Medium, CIRI + $20 \mathrm{mg} / \mathrm{kg}$ artesunate for 7 days group; High, CIRI + $40 \mathrm{mg} / \mathrm{kg}$ artesunate for $7 \mathrm{days}$ group. ${ }^{\# \#} \mathrm{P}<0.01$ vs. Sham; ${ }^{* *} \mathrm{P}<0.01$ vs. CIRI. Nrf2, nuclear factor erythroid 2-related factor 2; CIRI, cerebral ischemia/reperfusion injury; Bax, apoptosis regulator Bax; Bcl-2, apoptosis regulator Bcl-2; p, phosphorylated; MAPK, mitogen-activated protein kinase.

in patients with stroke and alleviate neuronal damage, thus improving the survival rate of stroke victims (22). However, following the restoration of the blood supply to ischemic tissues, the structure and function of cells undergo further damage caused by reperfusion, thus resulting in the development of CIRI (19). The results of the present study suggested that treatment with artesunate may prevent CIRI-induced neurological impairments, reduce the cerebral infarct volume and attenuate CIRI-associated histological damage in mice.

Oxidative stress results from an imbalance between oxidative and antioxidative processes, and may cause severe cell damage (8). An increase in the production of reactive oxygen and nitrogen species, and other free radicals, is the main cause of oxidative stress (23). ROS can interfere with gene expression regulation, thus altering the expression of signaling proteins, and affecting intracellular signaling cascades (24). Physiological ROS generation is critical for the maintenance of normal cellular functions; however, under pathological conditions, including cerebral ischemia, aberrant ROS production can result in the oxidation of cellular components, thus causing cell damage (25). In the present study, artesunate was demonstrated to significantly attenuate CIRI-induced impairments in the activity of the antioxidative enzymes SOD and $\mathrm{GSH}$ in mice.

Neurons are characterized by high metabolic activity and increased oxygen consumption. In addition, their relatively low endogenous antioxidant contents make neurons particularly sensitive to oxidative stress (26). Furthermore, the brain is rich in lipids, which can react with ROS to generate hydrogen peroxide free radicals, resulting in membrane lipid peroxidation and cell damage (27). Therefore, during CIRI, oxidative stress is among the main causes of neuronal injury. In the present study, artesunate was revealed to significantly inhibit ROS production in mice with CIRI. Cheng et al (14) suggested that artesunate may induce endothelial cell apoptosis through an ROS-dependent p38 MAPK/mitochondrial pathway. Conversely, the present findings suggested that the regulation of ROS production may be implicated in the protective effects of artesunate during CIRI.

Mitochondria are important organelles during the execution of apoptotic signals in neurons. Cerebral ischemia and reperfusion enhance the mitochondrial production of ROS, which, through the activation of the proapoptotic protein Bax, trigger the mitochondrial release of cytochrome $c$, which binds with apoptotic protease activating factor 1 and caspase-9 to form the apoptosome, resulting in the activation of caspase- 3 and other caspases, including caspase-2, -6, -8 and -10 (28). Activated caspase- 3 may subsequently cleave DNA repair enzymes, thus inhibiting the repair of ischemia-induced DNA damage, which results in cellular apoptosis (29). Oxidative stress and increased ROS production have been implicated in the development of CIRI (30). Under physiological conditions, low levels of ROS serve as signaling molecules in various cellular processes; however, in pathological states, when the endogenous antioxidative mechanisms are unable to counteract excessive ROS production, high levels of ROS can oxidize lipids, proteins, nucleic acids and intracellular components, thus causing cell damage (31). Direct or indirect protein 
oxidation by ROS can alter their structure, increase their hydrophobicity and interfere with protein-protein interactions, leading to the formation of protein aggregates, and causing cell damage (32). In the present study, artesunate was revealed to significantly suppress the activity of the proapoptotic caspase- 3 , reduce the $\mathrm{Bax} / \mathrm{Bcl}-2$ protein expression ratio and upregulate the expression of the antiapoptotic transcription factor Nrf2. Cao et al (16) reported that artesunate protected against sepsis-induced lung injury through the activation of Nrf 2 and heme oxygenase 1. Therefore, it may be hypothesized that increased $\mathrm{Nrf} 2$ expression participates in the suppression of ROS-induced apoptosis that is implicated in the protective effects of artesunate during CIRI.

CIRI-induced brain damage has been reported to involve several cellular processes, including increased production of oxygen free radicals and ROS-mediated injury, intracellular $\mathrm{Ca}^{2+}$ overload, cytokine-mediated injury, neurotoxicity caused by the aberrant release of excitatory amino acids, and disorders in neuronal metabolism (33). Inflammatory responses are implicated in the development of CIRI, and the MAPK signaling pathway has been reported to mediate inflammatory processes during CIRI (29). The inhibition of p38/MAPK signaling has been reported to reduce neuronal apoptosis in the rat hippocampal CA1 region during cerebral ischemia, thus indicating the implication of p38 MAPK activation in neuronal apoptosis (33). Following its activation via phosphorylation, p38 MAPK can activate downstream kinases and various transcription factors, and regulate the expression of target genes, including inducible nitric oxide synthase, TNF- $\alpha$ and IL-1 $\beta$ (34). These genes serve important roles in neuronal apoptosis and inflammatory responses during CIRI. The present results demonstrated that artesunate significantly suppressed the activation of the proinflammatory cytokines TNF- $\alpha$, IL- $1 \beta$ and IL-6, and enhanced the activity of anti-inflammatory IL-10 during CIRI, possibly through the suppression of $\mathrm{p} 38$ MAPK activation.

In conclusion, the results of the present study suggested that artesunate may protect against the development of CIRI, through the inhibition of oxidative and inflammatory processes, which may be regulated through the activation of Nrf2 and ROS-dependent p38 MAPK signaling pathways. Therefore, artesunate may have potential for the development of alternative therapeutic strategies aimed at the prevention and treatment of CIRI.

\section{References}

1. Sorond FA, Tan CO, LaRose S, Monk AD, Fichorova R, Ryan S and Lipsitz LA: Deferoxamine, cerebrovascular hemodynamics, and vascular aging: Potential role for hypoxia-inducible transcription factor-1-regulated pathways. Stroke 46: 2576-2583, 2015.

2. Kernan WN, Viscoli CM, Furie KL, Young LH, Inzucchi SE, Gorman M, Guarino PD, Lovejoy AM, Peduzzi PN, Conwit R, et al: Pioglitazone after ischemic stroke or transient ischemic attack. N Engl J Med 374: 1321-1331, 2016.

3. Huang X, Cheripelli BK, Lloyd SM, Kalladka D, Moreton FC, Siddiqui A, Ford I and Muir KW: Alteplase versus tenecteplase for thrombolysis after ischaemic stroke (ATTEST): A phase 2, randomised, open-label, blinded endpoint study. Lancet Neurol 14: 368-376, 2015 .

4. Liebeskind DS, Tomsick TA, Foster LD, Yeatts SD, Carrozzella J, Demchuk AM, Jovin TG, Khatri P, von Kummer R, Sugg RM, et al: Collaterals at angiography and outcomes in the Interventional Management of Stroke (IMS) III trial. Stroke 45: 759-764, 2014
5. Shan J, Sun L, Wang D and Li X: Comparison of the neuroprotective effects and recovery profiles of isoflurane, sevoflurane and desflurane as neurosurgical pre-conditioning on ischemia/reperfusion cerebral injury. Int J Clin Exp Pathol 8: 2001-2009, 2015.

6. Jiang F, Yang J, Zhang L, Li R, Zhuo L, Sun L and Zhao Q: Rosuvastatin reduces ischemia-reperfusion injury in patients with acute coronary syndrome treated with percutaneous coronary intervention. Clin Cardiol 37: 530-535, 2014.

7. Yu Q, Lu Z, Tao L, Yang L, Guo Y, Yang Y, Sun X and Ding Q: ROS-dependent neuroprotective effects of NaHS in ischemia brain injury involves the PARP/AIF pathway. Cell Physiol Biochem 36: 1539-1551, 2015.

8. Zhao B, Chen Y, Sun X, Zhou M, Ding J, Zhan JJ and Guo LJ: Phenolic alkaloids from Menispermum dauricum rhizome protect against brain ischemia injury via regulation of GLT-1, EAAC1 and ROS generation. Molecules 17: 2725-2737, 2012.

9. Wang PR, Wang JS, Zhang C, Song XF, Tian N and Kong LY: Huang-Lian-Jie-Du-Decotion induced protective autophagy against the injury of cerebral ischemia/reperfusion via MAPK-mTOR signaling pathway. J Ethnopharmacol 149: 270-280, 2013.

10. Cheng CY, Lin JG, Tang NY, Kao ST and Hsieh CL: Electroacupuncture at different frequencies $(5 \mathrm{~Hz}$ and $25 \mathrm{~Hz}$ ) ameliorates cerebral ischemia-reperfusion injury in rats: Possible involvement of p38 MAPK-mediated anti-apoptotic signaling pathways. BMC Complement Altern Med 15: 241, 2015.

11. Wang S, Liu K, Seneviratne CJ, Li X, Cheung GS, Jin L, Chu CH and Zhang C: Lipoteichoic acid from an Enterococcus faecalis clinical strain promotes TNF- $\alpha$ expression through the NF- $\kappa B$ and p38 MAPK signaling pathways in differentiated THP-1 macrophages. Biomed Rep 3: 697-702, 2015.

12. Nito C, Kamada H, Endo H, Niizuma K, Myer DJ and Chan PH: Role of the p38 mitogen-activated protein kinase/cytosolic phospholipase A2 signaling pathway in blood-brain barrier disruption after focal cerebral ischemia and reperfusion. J Cereb Blood Flow Metab 28: 1686-1696, 2008.

13. Jie P, Hong Z, Tian Y, Li Y, Lin L, Zhou L, Du Y, Chen L and Chen L: Activation of transient receptor potential vanilloid 4 induces apoptosis in hippocampus through downregulating PI3K/Akt and upregulating p38 MAPK signaling pathways. Cell Death Dis 6: e1775, 2015

14. Cheng R, Li C, Li C, Wei L, Li L, Zhang Y, Yao Y, Gu X, Cai W, Yang $\mathrm{Z}$, et al: The artemisinin derivative artesunate inhibits corneal neovascularization by inducing ROS-dependent apoptosis in vascular endothelial cells. Invest Ophthalmol Vis Sci 54: 3400-3409, 2013.

15. Thanaketpaisarn O, Waiwut P, Sakurai H and Saiki I: Artesunate enhances TRAIL-induced apoptosis in human cervical carcinoma cells through inhibition of the $\mathrm{NF}-\kappa \mathrm{B}$ and PI3K/Akt signaling pathways. Int J Oncol 39: 279-285, 2011.

16. Cao TH, Jin SG, Fei DS, Kang K, Jiang L, Lian ZY, Pan SH, Zhao MR and Zhao MY: Artesunate protects against sepsis-induced lung injury via heme oxygenase-1 modulation. Inflammation 39: 651-662, 2016.

17. Ng DS, Liao W, Tan WS, Chan TK, Loh XY and Wong WS: Anti-malarial drug artesunate protects against cigarette smoke-induced lung injury in mice. Phytomedicine 21: 1638-1644, 2014.

18. Wu F, Li J, Guo N, Wang XH and Liao YQ: MiRNA-27a promotes the proliferation and invasion of human gastric cancer MGC803 cells by targeting SFRP1 via Wnt/ $\beta$-catenin signaling pathway. Am J Cancer Res 7: 405-416, 2017.

19. Svensson LG, Blackstone EH, Apperson-Hansen C, Ruggieri PM, Ainkaran P, Naugle RI, Lima B, Roselli EE, Cooper M, Somogyi D, et al: Implications from neurologic assessment of brain protection for total arch replacement from a randomized trial. J Thorac Cardiovasc Surg 150: 1140-1147.e11, 2015.

20. Yuan Y, Yao YF, Hu SN, Gao J and Zhang LL: MiR-133a is functionally involved in doxorubicin-resistance in breast cancer cells MCF-7 via its regulation of the expression of uncoupling protein 2. PLoS One 10: e0129843, 2015.

21. Fernandez-Twinn DS, Blackmore HL, Siggens L, Giussani DA, Cross CM, Foo R and Ozanne SE: The programming of cardiac hypertrophy in the offspring by maternal obesity is associated with hyperinsulinemia, AKT, ERK, and mTOR activation. Endocrinology 153: 5961-5971, 2012. 
22. Jones KM, Bhattacharjee R, Krishnamurthi R, Blanton S, Theadom A, Barker-Collo S, Thrift A, Parmar P, Maujean A, Ranta A, et al: Methodology of the stroke self-management rehabilitation trial: An international, multisite pilot trial. J Stroke Cerebrovasc Dis 24: 297-303, 2015.

23. Saad MA, Abdelsalam RM, Kenawy SA and Attia AS: Ischemic preconditioning and postconditioning alleviates hippocampal tissue damage through abrogation of apoptosis modulated by oxidative stress and inflammation during transient global cerebral ischemia-reperfusion in rats. Chem Biol Interact 232: 21-29, 2015.

24. Shen MH, Zhang CB, Zhang JH and Li PF: Electroacupuncture attenuates cerebral ischemia and reperfusion injury in middle cerebral artery occlusion of rat via modulation of apoptosis, inflammation, oxidative stress, and excitotoxicity. Evid Based Complement Alternat Med 2016: 9438650, 2016

25. Sosunov SA, Ameer X, Niatsetskaya ZV, Utkina-Sosunova I, Ratner VI and Ten VS: Isoflurane anesthesia initiated at the onset of reperfusion attenuates oxidative and hypoxic-ischemic brain injury. PLoS One 10: e0120456, 2015.

26. Buch P, Patel V, Ranpariya V, Sheth N and Parmar S: Neuroprotective activityof Cymbopogon martinii against cerebral ischemia/reperfusion-induced oxidative stress in rats. J Ethnopharmacol 142: 35-40, 2012.

27. Palencia G, Medrano JÁ, Ortiz-Plata A, Farfán DJ, Sotelo J, Sánchez A and Trejo-Solís C: Anti-apoptotic, anti-oxidant, and anti-inflammatory effects of thalidomide on cerebral ischemia/reperfusion injury in rats. J Neurol Sci 351 78-87, 2015.

28. Zhang M, Su L, Xiao Z, Liu X and Liu X: Methyl jasmonate induces apoptosis and pro-apoptotic autophagy via the ROS pathway in human non-small cell lung cancer. Am J Cancer Res 6: 187-199, 2016.
29. Zhou L, Chen L, Wang J and Deng Y: Astragalus polysaccharide improves cardiac function in doxorubicin-induced cardiomyopathy through ROS-p38 signaling. Int J Clin Exp Med 8: 21839-21848, 2015

30. Yong Y, Matthew S, Wittwer J, Medrano JÁ, Ortiz-Plata A, Farfán DJ, Sotelo J, Sánchez A and Trejo-Solís C: Dichamanetin inhibits cancer cell growth by affecting ROS-related signaling components through mitochondrial-mediated apoptosis. Anticancer Res 33: 5349-5355, 2013.

31. Lin H, Gao X, Chen G, Sun J, Chu J, Jing K, Li P, Zeng R and Wei B: Indole-3-carbinol as inhibitors of glucocorticoid-induced apoptosis in osteoblastic cells through blocking ROS-mediated Nrf2 pathway. Biochem Biophys Res Commun 460: 422-427, 2015.

32. Lucas IK and Kolodziej H: Trans-resveratrol induces apoptosis through ROS-triggered mitochondria-dependent pathways in A549 human lung adenocarcinoma epithelial cells. Planta Med 81: 1038-1044, 2015

33. Jiang M, Li J, Peng Q, Sun J, Chu J, Jing K, Li P, Zeng R and Wei B: Neuroprotective effects of bilobalide on cerebral ischemia and reperfusion injury are associated with inhibition of pro-inflammatory mediator production and down-regulation of JNK1/2 and p38 MAPK activation. J Neuroinflammation 11: 167, 2014.

34. Qi SH, Hao LY, Yue J, Zong YY and Zhang GY: Exogenous nitric oxide negatively regulates the S-nitrosylation p38 mitogen-activated protein kinase activation during cerebral ischaemia and reperfusion. Neuropathol Appl Neurobiol 39: 284-297, 2013. 